\title{
POPULATION SYNTHESIS STUDIES OF WHITE DWARF BINARIES
}

\author{
U. Kolb ${ }^{1}$ and B. Willems ${ }^{2}$ \\ RESUMEN
}

Presentamos estudios de síntesis de población de binarias enana blanca - estrella de la secuencia principal. de variables cataclísmicas que son conducidas por discos circumbinarios y de binarias eclipsantes. en la búsqueda del tránsito de exoplanetas SuperWASP.

\section{ABSTRACT}

We present population synthesis studies of white dwarf - main-sequence star binaries, of cataclysmic variables that are driven by circumbinary discs, and of eclipsing binaries in the exoplanet transit search SuperWASP.

Key Words: BINARIES: CLOSE — BINARIES: ECLIPSING - WHITE DWARFS

\section{INTRODUCTION}

We use the BiSEPS population synthesis code developed by Willems \& Kolb (2002) to study binaries with a white dwarf component. The code makes use of the analytic fits to the evolution of single stars by Hurley et al. (2000), and follows largely the scheme to treat the evolution of binary stars proposed by Hurley et al. (2002), but was independently developed. For details see Willems \& Kolb (2002).

\section{WHITE DWARF - MAIN-SEQUENCE STAR BINARIES}

Willems \& Kolb (2004) revisited the population of Galactic white dwarf (WD) main-sequence (MS) star binaries. Seven evolutionary channels have been identified, as summarised in Table 1. The comprehensive population synthesis serves as a basis for more detailed studies of subclasses and binaries that descend from WD-MS star binaries.

The ratio of systems that contain a helium WD to those that contain a $\mathrm{C} / \mathrm{O}$ or $\mathrm{O} / \mathrm{Ne} W D$ may act as a useful diagnostic for constraining the common envelope ejection efficiency $\alpha_{C E}$ (Fig. 1). If $\alpha_{C E}<1$ this ratio is sensitive to $\alpha_{C E}$ but insensitive to the shape of the otherwise little constrained initial mass ratio distribution $n(q)$. For larger $\alpha_{C E}$ the ratio of WD types is affected by both $\alpha_{C E}$ and $n(q)$.

The observed WD type ratio will depend on observational selection effects. One of these is determined by the luminosity difference between the WD and its MS star companion. As can be seen in Fig. 2 the WD type ratio varies with the limiting luminos-

\footnotetext{
${ }^{1}$ Dept. of Physics and Astronomy, The Open University, Milton Keynes, UK.

${ }^{2}$ Department of Physics and Astronomy, Northwestern University, Evanston, USA.
}

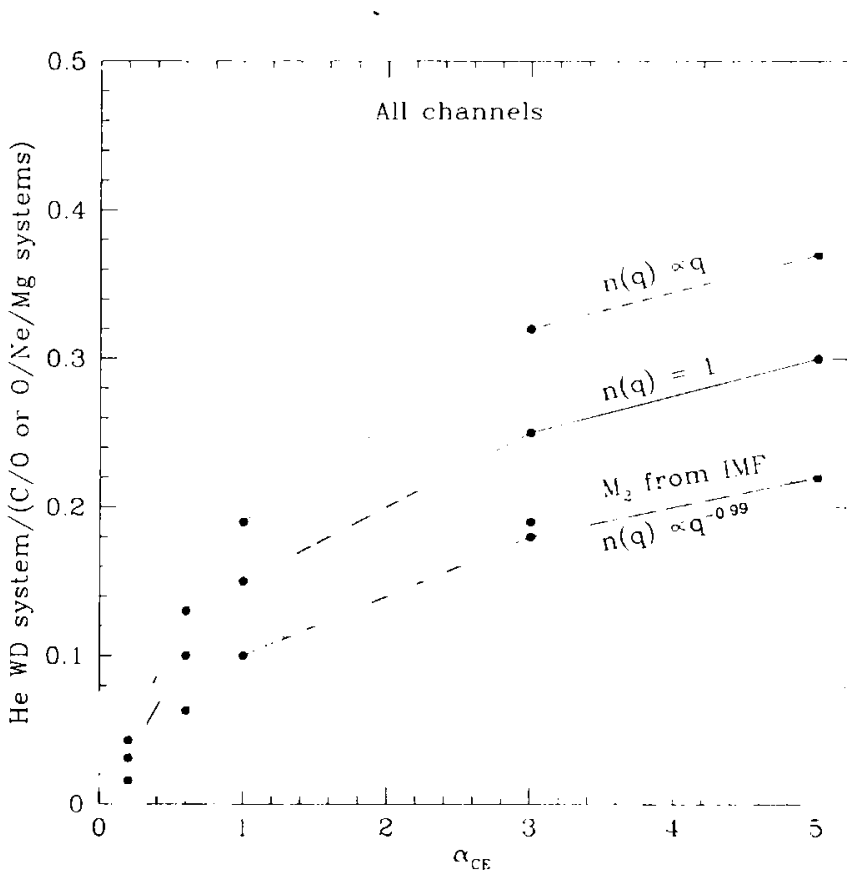

Fig. 1. Ratio of He white dwarf - MS star binaries to $\mathrm{C} / \mathrm{O}$ or $\mathrm{O} / \mathrm{Ne}$ WD-MS star binaries for different initial mass ratio or initial secondary mass distributions, as a function of the common-envelope ejection efficiency $\alpha_{C E}$ for WDMS binaries forming through all considered formation channels.

ity ratio $L_{\| D} / L_{M S}$ that is necessary for a positive detection of the WD.

For more details we refer to Willems \& holb (2004).

\section{CIRCUMBINARY-DISC DRIVEN EVOLUTION OF CIS}

Spruit \& Taam (2000) suggested circumbinary discs as a possible driver of cataclysmic variable (CV) evolution. Taam te al. (20)33) presented the most refined numerical models of the evolution of 


\section{TABLF; 1}

\section{FORMATION C'HANNELS OF WD-MS STAR BINARIES}

\begin{tabular}{|c|c|c|c|c|}
\hline Channel & nature of 1st RLOF & description & remnant WD & $\%$ \\
\hline 1 & stable & Case A/B & $\mathrm{He}$ & $5-15$ \\
\hline 2 & stable & Case $\mathrm{B}, \mathrm{nHe}$ & $\mathrm{C} / \mathrm{O}, \mathrm{O} / \mathrm{Ne}$ & $0.1-4$ \\
\hline 3 & stable & Case (! & $\mathrm{C} / \mathrm{O}, \mathrm{O} / \mathrm{Ne}$ & $0.1-3$ \\
\hline 4 & unstablo & Casc B CE & $\mathrm{He}$ & $35-40$ \\
\hline 5 & unstable? & Case B CH. nHe & $\mathrm{C} / \mathrm{O}, \mathrm{O} / \mathrm{Ne}$ & 0.1 \\
\hline 6 & unstiable? & Case C: CE & $\mathrm{C} / \mathrm{O}, \mathrm{O} / \mathrm{Ne}$ & $50-55$ \\
\hline 7 & non-interacting & - & $\mathrm{C} / \mathrm{O}, \mathrm{O} / \mathrm{Ne}$ & $\mathrm{C} 7:(\mathrm{C} 1-\mathrm{C} 6)=7: 3$ \\
\hline
\end{tabular}

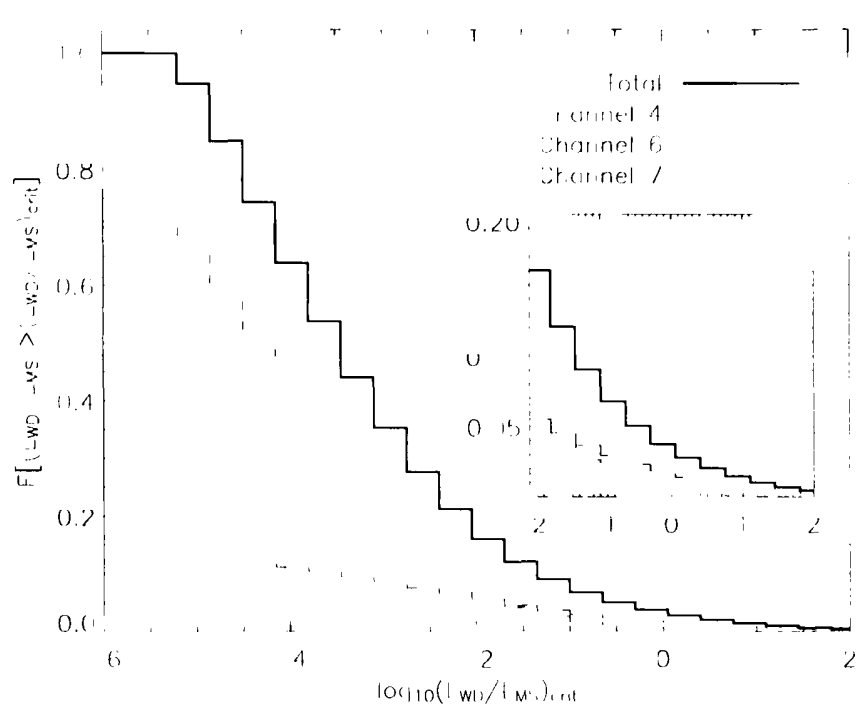

Fig. 2. The fraction $F$ of systems for which the WD to MS' star luminosity ratio is larger than the limiting ratio $\left(L_{W D} / L_{\Lambda s}\right)_{\text {. it }}$, in the casse of a standard population synthesis model (sere Willems \& Kolb 2004 for details). The thick solid line represents the total for all systems forming through formation channels 17 , while the thin lines distinguish bot ween the contributions of chamnels 4. 6 and 7 .

CVs with circumbinary dises. In the light of continuing doubts of the validity of the standard disrupted magnetic braking model for the ( $\mathrm{V}$ period gap (e.g. Andronov et. al. 2003) wo investigate if circumbinary disc-driven evolution can lead to a feature reminiscent of the period gap in the $(\mathrm{V}$ orbital period distribution. To do so we assume an ad-hoc continuous magnetic braking with a timescale of $10^{10} \mathrm{mr}$. while other parameters affecting the formation and evolution of CVs (such as $(x)$ and $n(q)$ ) are varied.

We make use of an extended grid of circumbinary disc-driven erolutionary sequences of (Cls. a subset of which was published by Taam of al. (2003). The disc feeding parameter $\dot{\delta}$. the mass accretion rate into the circumbinary disc in units of the mass

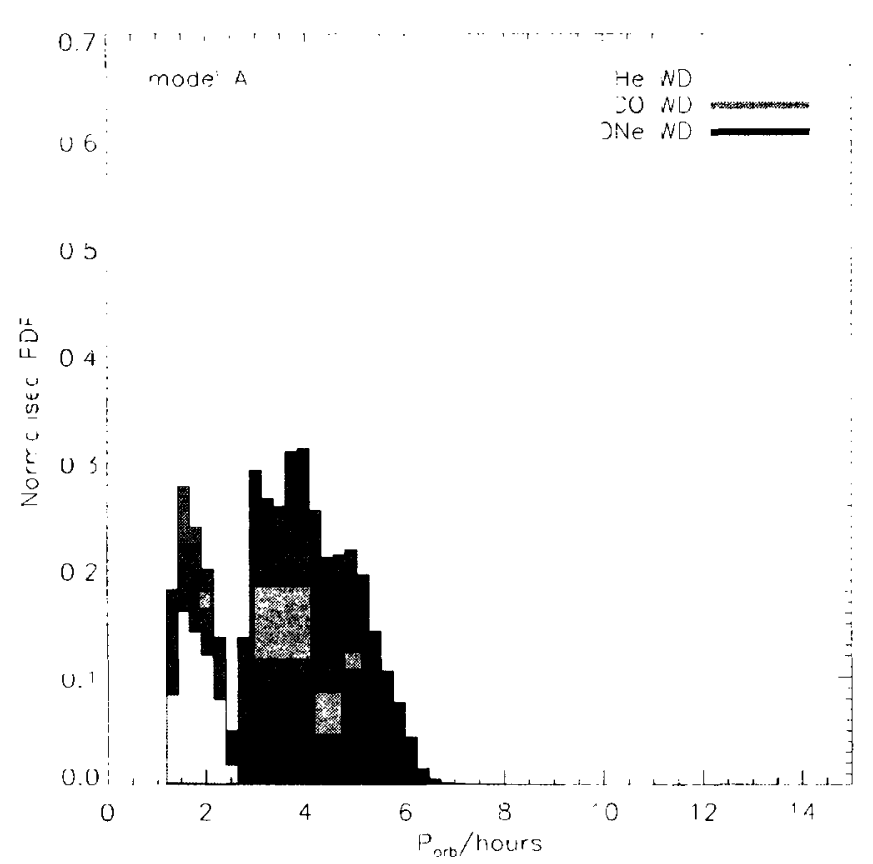

Fig. 3. Predicted orbital period distributions of $\mathrm{CVs}$ that evolve under the influence of a circumbinary disc.

transfer rate, is assumed to be bimodal $(\delta=0.0003$ for donor star mass $M_{2}>0.35 M_{\odot}, \delta=0.0001$ for $\left.M_{2}<0.35 M_{\odot}\right)$.

Figure 3 shows the resulting orbital period distribution of a complete, volume-limited sample of $\mathrm{CVs}$, for standard parameters as in Willems \& Kolb (2002).

We find that a gap feature does appear only if $\delta$ is bimodal, and that in the intrinsic population the number of systems above and below the period gap region is about equal. Any likely observational selection effect would favour the brighter systems above the gap. so in order to reproduce the observed period distribution (e.g. Ritter \& Kolb 2003) the intrinsic distribution should have a lot more power at short orbital periods. We attempted and failed to achieve this by varying standard parameters within reason- 


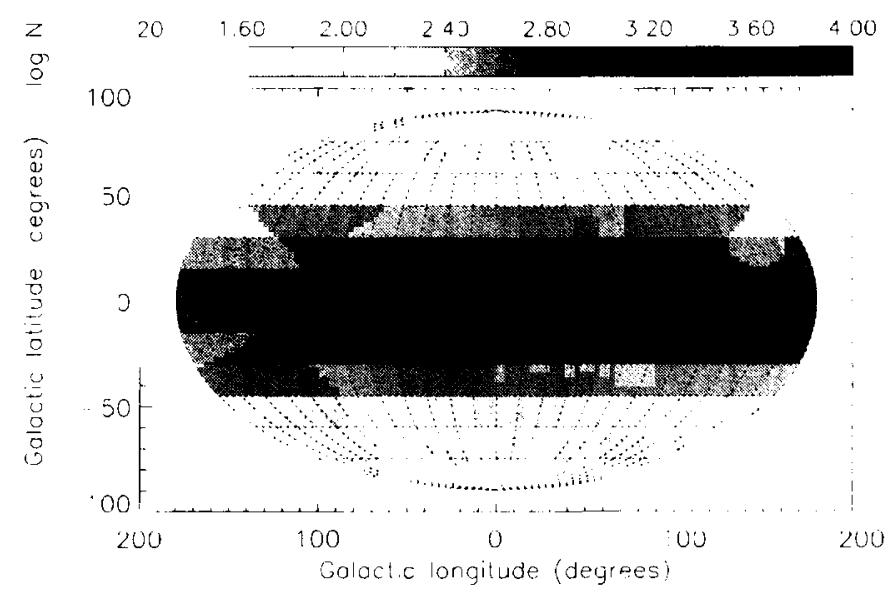

Fig. 4. Detectable number $\mathrm{N}$ of eclipsing binaries per $15^{\circ} \times 15^{\circ}$ field ( $\mathrm{N}$ is on a log scale).

able limits. This may make circumbinary discs a less attractive model for explaining the CV period gap. A full account of our numerical experiments will be published elsewhere (Willems et al. 2004, in preparation).

\section{ECLIPSING BINARIES IN EXOPLANET TRANSIT SEARCHES: SUPERWASP}

Exoplanet transit searches will inevitably also detect a large number of eclipsing binaries (EBs). The systematic nature and well-defined selection effects of some of the search projects will deliver a sample of EBs that lends itself to exploitation by population synthesis techniques.

We present preliminary calculations for the expected EB detection rate of SuperWASP (Kane et al. 2003, Street et al. 2003; see also http://www.superwasp.org), a wide-angle angle search for planets which is sensitive to transits with an amplitude in excess of $0.01 \mathrm{mag}$ in the magnitude range $7<m_{V}<13$ in a combined field of view of about $15^{\circ} \times 15^{\circ}$. The first of several SuperWASP camera sets has just become operational.

We used the Galactic absorption model by Hakkila et al. (1997) to calculate the distribution of EBs as a function of Galactic latitude and longitude. For standard population synthesis parameters the resulting number of EBs per field of view are shown in Figs. 4 and 5, both in absolute terms and in units of the number of detectable single stars in the same field of view.

Using standard population parameters our simulations predict that SuperWASP should find about $5 \times 10^{5}$ EBs among a total of about $7 \times 10^{6}$ stars; up to $7 \%$ of stars in a SuperWASP field are expected to be EBs. The table spells out the expected frequency of major binary combinations.

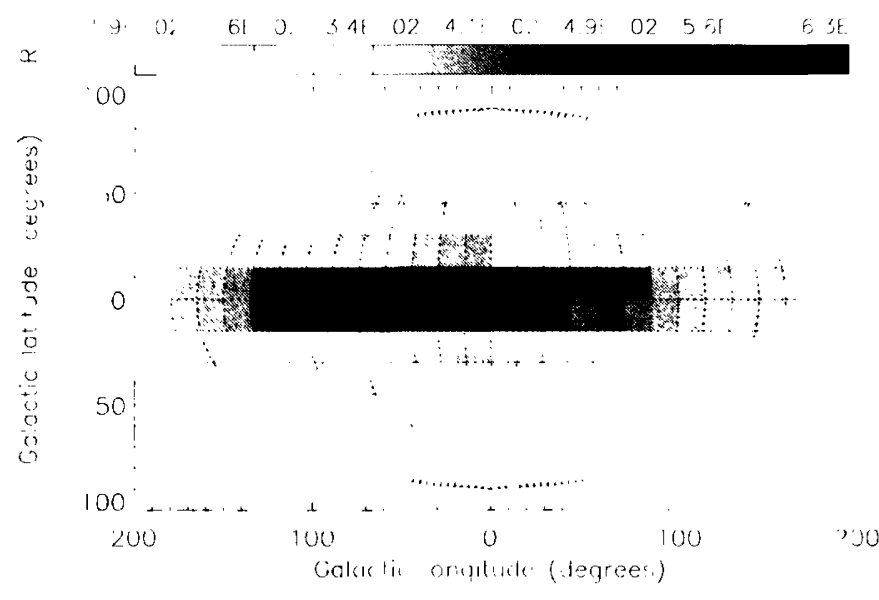

Fig. 5. Same as previous figure, but in units of the number of detectable single stars per ficld (linear scale).

TABLE 2

\section{ECLIPSING BINARIES DETECTABLE BY} SUPER-WASP

\begin{tabular}{llll}
\hline EB type & fraction & EB typo & fraction \\
\hline MS+MS & $11 \%$ & AGB+MS & $2: 3 \%$ \\
HG+MS & $15 \%$ & X + MS RLOF & $11 \%$ \\
GB +MS & $16 \%$ & X + WD & $1 \%$ \\
HB +MS & $11 \%$ & & \\
\hline
\end{tabular}

For a detailed account of assumptions and the technique used to calculate the distributions shown on the figures, see Willems, Kolb \& Justhan 20)(4. forthcoming.

This research was supported by PPARC.

\section{REFERENCES}

Andronov N., Pinnsoneault M., Sills A. 2003, 582, 358 Hakkila J., Myers J.M., Stidham B..J., Hartmann D.H., 1997, A.J 114, 2042

Hurley J.R., Pols O.R., Tout C.A. 2000, MNRAS 315. 543

Hurley J.R., Tout C.A., Pols O.R. 2002, MNRAS 329. 897

Kane S.R., Horne K., Strert R.A., Pollacco D.L., James D., Tsapras Y., Collier Cameron A., 2003, to be published in the PASP proceedings of "Scientific Frontiers in Research on Extrasolar Planets"

Ritter H., Kolb U. 2003, A\& A, 10.1. 301

Spruit H.C., Taam R.E., 2000, ApJ, 5-18. 900

Street R.A. et al., 2003, to be published in the P.ASP proceedings of "Scientific Frontiers in Research on Extratsolar Planets".

Taam, R.E., Sandquist, E.L., Dubus G., 2003, ApJ. 59)2. i 124

Willerns, B., \& Kolb, L' 2002, MNIR iS 337. 1004 2004, A\& A in press 